Constanze Neupetsch*, Eric Hensel, Michael Werner, Sven Meißner, Jan Troge, Welf-Guntram Drossel, and Christian Rotsch

\title{
Development and Validation of Bone Models using Structural Dynamic Measurement Methods
}

https://doi.org/10.1515/cdbme-2019-0086

\begin{abstract}
Vibration measurement and signal analysis methods are common to evaluate the functionality and characteristics of technical components in different industrial and scientific areas. Modal analysis for example is a standard method to characterize the dynamic behavior of a structure and enables the development of validated bone models. The state of the art of analyzing bone structures does not include the modal damping, although it has a significant influence on the dynamic characteristics. Within the presented investigations, the modal analyses have been performed contactless with respect to excitation and response acquisition, which implies that there are no influences of shakers or sensor couplings. Therefore, an automatic impulse hammer and a 3D Scanning Laser Doppler Vibrometer were used for excitation and response detection. Various supports of the test specimens, surface pretreatments, excitation points and excitation impulses were examined to optimize the measurement setup and process. Experimental modal analysis data were analyzed by curve fitting methods to determine the modal parameters. To evaluate different structures and effects of damp ing, 3D printed artificial bones and animal in vitro bones were used to perform the measurements. To produce the cortical layer of the artificial bone models, volume models were generated based on medical image data and printed by polyamide-based selective laser sintering. The cancellous bone was represented by different foam fillings for the artificial bones. Thereby, the variation of the porosity was achieved by using different mixing ratios of polyurethane foam and hardener. Furthermore, the modal damping parameters were determined from the measurement of animal bones. The measurement time was optimized during the practical implementation of the parameter determination to minimize the influence of drying and decomposition processes on the measurement results.
\end{abstract}

${ }^{*}$ Corresponding author: Constanze Neupetsch, Chemnitz University of Technology, Professorship for Adaptronics and Lightweight Design in Production, Chemnitz, Germany, e-mail: constanze.neupetsch@mb.tu-chemnitz.de

Eric Hensel, Michael Werner, Sven Meißner, Jan Troge, WelfGuntram Drossel, Christian Rotsch, Fraunhofer Institute for Machine Tools and Forming Technology IWU, Chemnitz/Dresden, Germany
Keywords: Experimental Modal Analysis, Bone Structure, Artificial Bone, 3D Scanning Laser Doppler Vibrometry

\section{Introduction}

Simulations of a surgery on human specimens offer the opportunity to practice complex procedures in the advanced training of physicians. However, human specimens are only available for this purpose to a limited extent and different comorbidities of the donors have to be considered. Artificial training models offer a more cost-effective and reproducible alternative and can also be used for functional biomechanical testing, for example the development of new implants. However, the realism of representation, especially of bony structures, is very limited and measurements do not provide the same results compared to human specimens. For example, the occurring fractures are not comparable (cf. [1]) and in particular, the elastic properties and strength of common bone simulant material are described as insufficient [2]. Advanced models, such as the customized bone models of the distal femur of Wähnert et al. [3] or the 3D-printed spine models of Hao et al. [4] do not provide satisfactory results at present.

The objective for the future is to develop realistic bone models as a base for improved surgical training models to enhance the medical education. Further, bone models with correct biomechanical properties can improve and accelerate the development processes for new medical devices and technologies. The first step was the determination of the biomechanical parameters of human bones to generate reproducible artificial bone models. The aim of the present study is the development of a procedure to characterize and validate bone models using structural dynamic measurement methods. Conventional mechanical tests for the characterization of material properties require special specimen geometry. However, these can only be achieved to a limited extent with bone tissue. An alternative possibility for material characterization is the comparison of experimentally and numerically determined modal parameters such as eigenfrequencies, damping and vibration modes [5]. 


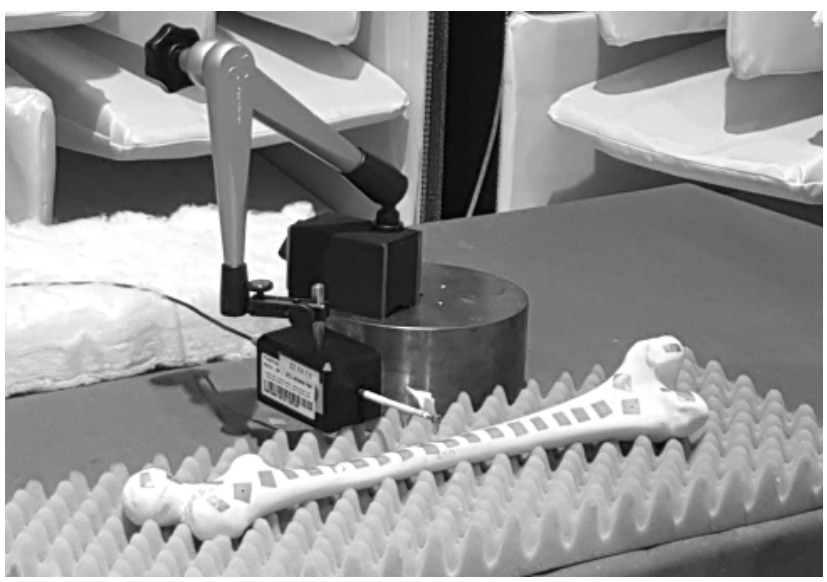

Fig. 1: Test setup of the EMA of synthetic bones supported on insulation material.

\section{Test Setup, Data Acquisition and Processing}

As mentioned above, the dynamic characteristics of the various bone models are obtained by experimental modal analyses (EMA). There are two different challenges to deal within an EMA in general. At first, the test object has to be excited in a defined way, which is realized by impact hammers or shakers in most cases. Both types hold their own advantages and drawbacks. The main disadvantage of an electrodynamic exciter is the connection of shaker and structure which is usually realized with an impedance or force sensor to obtain the induced force. The use of an impact hammer enables the excitation of the structure without the possibility of affecting its dynamic properties. On the other hand, the excitation via shaker allows the use of different signal waveforms (e.g. sweep or random) where the impact hammer measurement is limited to impulselike force-time-histories. The second challenge within an EMA is the selection of the boundary condition (BC) and the support, respectively. The two ideal $\mathrm{BC}$ are represented by a fixed or free support of the structure, again, with their own advantages and drawbacks. The fixed BC can be realized, for example, by gluing the test structure on a rigid body (rigid in comparison to the test structure). In analogy to the shaker excitation, the connection of the test object to the rigid support can influence the dynamic properties of the structure. In addition, the connection between test object and support may vary, which can lead to statistical deviations for repeating measurements.

Due to the described advantages and disadvantages, a test setup according to Figure 1 has been chosen. As shown in the figure, the excitation of the various bones have been carried out by an automatic impact hammer (MAUL-THEET GmbH, type: vImpact-60) to ensure uniform excitations over the entire measurements. The bones are supported by an elastic insulation material. Of course, the insulation material can affect the modal characteristics of the test object. Due to that fact, additional measurements under idealized free/free BC (bones supported by elastic cords) has been carried out to evaluate the effect of the support, for example, on the modal damping of the bones. The advantage of the insulation material is based on the lower rigid body movement in comparison to the idealized free/free BC. This enables shorter overall measurement times which are necessary due to the dehydration of biological bones. The dehydration leads to a modification of the bone's structure and thus, it can affect the dynamic properties. The evaluation of the insulation material's effect on the modal damping of the test structures will be content of section 3 .

Another important issue in terms of EMA is the way of acquiring the response of the test structure. In the present case, the vibrations of the bones are obtained by optical measurements using a 3D Scanning Laser Doppler Vibrometer (Polytec GmbH, type: $P S V-400-3 D$ ). The advantage of the optical data acquisition is, in analogy to the excitation via impact hammer, that the test object is not influenced by a sensor's mass or its application. The optical measurements with the above mentioned system require a special surface treatment to ensure a sufficient quality of the laser beam reflected by the vibrating surface. The pretreatment can be realized using a crack detection spray or applying a retroreflective tape. The use of retroreflective tapes enables the best signal-to-noise ratio but requires more time in preparation which is an important point due to the addressed dehydration. Section 3 includes the corresponding comparison of different surface pretreatments. The problem of dehydration has been introduced as a special issue within the EMA of biological bones. Another important topic is the definition of a uniform coordinate system for all samples. In addition to the modal analyses described within this paper, the bones are scanned by computed tomography (CT) to analyze the corresponding inner structure and to generate exact finite element models. Thus, uniform coordinate systems are necessary. A subsequent transformation of coordinate systems is possible but constitutes additional error sources. Figure 2 shows three markers which have been applied to the structure (sheep bone).

Within the EMA, excitation forces and surface velocities have been acquired based on the used measurement equipment described above. The obtained frequency response functions have been post-processed in a subsequent step using so-called curve fitting methods to obtain the resultant modal parameters eigenfrequencies, modal damping and mode shapes from the experimental data.

For each measured structure, five repeating measurements have been carried out to evaluate the corresponding statistical certainty. 


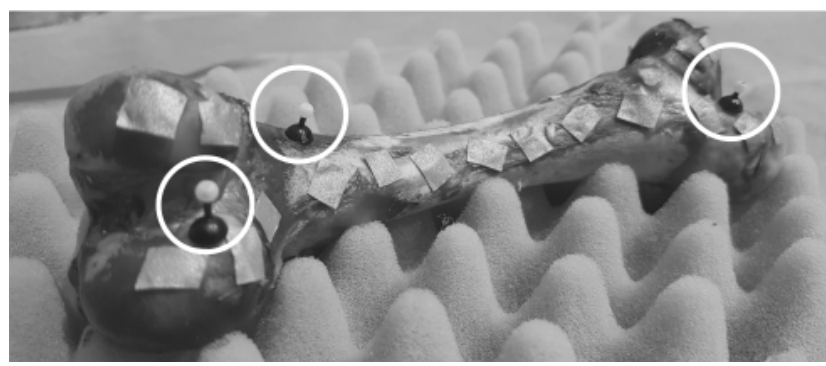

Fig. 2: Test setup of the EMA of sheep bone (highlighted markers).

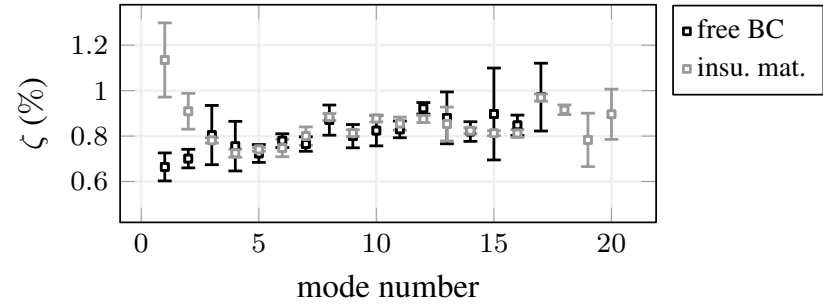

Fig. 3: Error bar diagram of damping ratios in dependency of corresponding mode shape number (error bar interval $\pm 2 \sigma$ ).

\section{Results}

At first, the influence of the insulation material on the modal damping is described. Figure 3 shows the corresponding comparison of damping ratios $\zeta$ (percent of critical damping) of the idealized free/free BC and the support on the insulation material. The diagram displays the damping ratios and the corresponding error bar interval in dependency on the mode shape number. The standard deviations have been calculated for five repeating measurements of each setup and for each mode where the equality of mode shapes has been ensured by modal assurance criterion values greater than $80 \%$. At first, Figure 3 shows low deviations of the determined damping ratios for both setups. Furthermore, it is noticeable that the insulation material only affects the modal damping values of the first two modes which can be identified as the first two bending modes at $112.9 \mathrm{~Hz}$ and $137.5 \mathrm{~Hz}$ of the investigated synthetic bone. For the remaining displayed modes in a frequency range up to $\approx 4.6 \mathrm{kHz}\left(20^{\text {th }}\right.$ mode shape of the measurement with insulation material), no influence of the support can be detected. For further evaluations, the damping ratios of the first two bending modes can be corrected using the displayed differences.

In a second step, three different synthetic bones have been analyzed. These three bone models share the same outer geometry but differ in their inner structure resulting from various foam densities (weights: $m_{\mathrm{SB} 000}=105.9 \mathrm{~g}, m_{\mathrm{SB} 200}=142.7 \mathrm{~g}$, $m_{\mathrm{SB} 650}=247.0 \mathrm{~g}$, volume: $V_{i}=295.8 \mathrm{~cm}^{3}$ ). An objective of the measurement of these three different synthetic bones

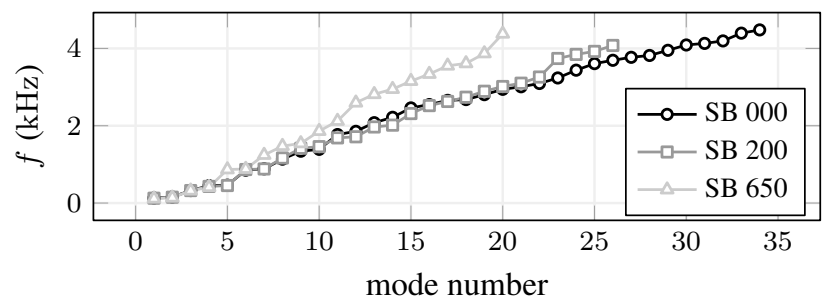

Fig. 4: Eigenfrequencies of different synthetic bones.

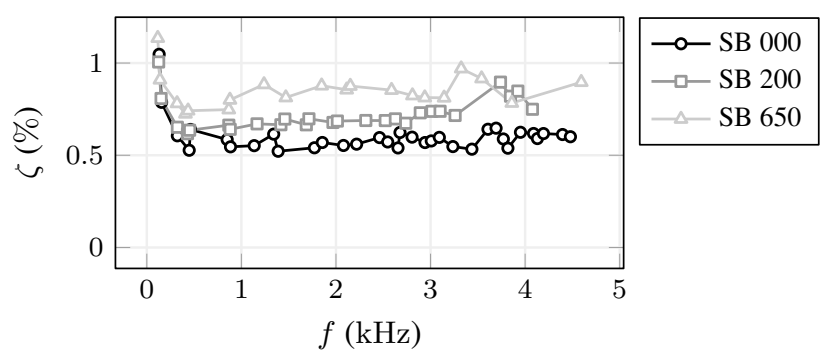

Fig. 5: Damping ratios of different synthetic bones.

is to find out how the modal parameters will change due to the modifications and furthermore, in which ranges they will change. Figures 4 and 5 show the obtained eigenfrequencies in dependency of the corresponding mode shape number. The eigenfrequency curves point out that there are significant differences between the various synthetic bones especially at higher frequencies. The largest gap can be found for SB 650 which is reasonable due to the difference in density in comparison to the two remaining structures. Because of the higher mass of SB 650 the increase of eigenfrequencies is caused by a larger stiffness compared to SB 000 and SB 200. Furthermore, an impact on the obtained damping ratios can be detected which is represented by Figure 5. The curves of eigenfrequencies and damping ratios show that the modal parameters of synthetic bones can be influenced significantly by modification of the inner structure using different foam densities in the present case. Within the underlying research project, synthetic bones have to be developed which represent the mechanical properties of human bones. Thus, the shown preinvestigations provide a basis for the modification of artificial bones to ensure the optimal representation of biological (human) structures.

Finally, first measurements of biological materials are presented below. So far, two different biological bones have been investigated: a femur of a pork and a femur of a sheep. The main objective is to ensure the approach of an optimized measurement flow described above (overall time reduction due to dehydration etc.). At first, the influence of the surface pretreatment is analyzed. Since the surfaces of the synthetic bones and the biological ones are not comparable, the investigation of the pretreatment is carried out using biological structures. The evaluation of the different surface pretreatments described in 


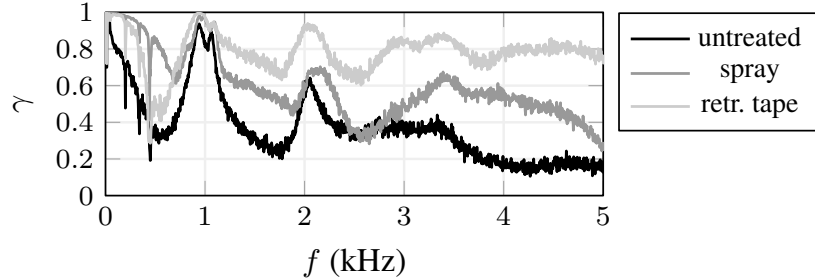

Fig. 6: Coherences for surface pretreatments (pork femur).

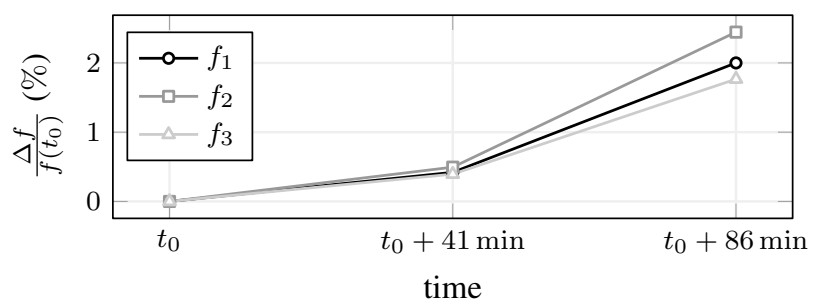

Fig. 7: Time-dependent change of first three eigenfrequencies of the sheep femur.

section 2 is based on the averaged coherence spectra which are displayed up to $5 \mathrm{kHz}$ in Figure 6. It can be seen that the surface pretreatment with retroreflective tape leads to significant better coherence values within the considered frequency range. Due to this improvement in comparison to the remaining two treatments, further investigations are carried out using the retroreflective tape. The application of the small pieces of retroreflective tape can be seen in the Figures 1 and 2.

Finally, the results of the EMA of the sheep femur shall be discussed. In comparison to the synthetic bones, dehydration can occur for biological materials. Due to this fact, three different measurements have been carried out at a time $t_{0}$ (as reference) as well as $41 \mathrm{~min}$ and $86 \mathrm{~min}$ later with respect to $t_{0}$ (indicated with $t_{0}+41 \mathrm{~min}$ and $t_{0}+86 \mathrm{~min}$ ). Figure 7 represents the time-dependent change of the first three eigenfrequencies: the first bending mode $f_{1}$ at $1.24 \mathrm{kHz}$, the second bending mode $f_{2}$ at $1.34 \mathrm{kHz}$ and the first torsional mode $f_{3}$ at $1.59 \mathrm{kHz}$. The increasing values clarify the problem of the dehydration process since all eigenfrequencies increase up to $\approx 2 \%$ regarding to $t_{0}$. Thus, a short measurement time is necessary to minimize the influence of dehydration. The time-dependent change of the modal damping is not displayed here but shows a non-uniform change of the damping ratios of the single modes and can not be analyzed in detail due to the missing statistical evaluation. This variation of modal damping in terms of dehydration is content of further steps within the corresponding research project. In comparison to the synthetic bones, significant differences can be detected for the obtained damping ratios. However, final comparisons should be carried out with human bones to get exact deviations between human and artificial bones.

\section{Conclusion and Outlook}

Within the present paper, experimental modal analyses of artificial and biological bones have been carried out. Initially, the challenges in terms of modal analyses using contactless excitation and response acquisition are discussed for bone measurements. Under consideration of the described issues, the results of different preinvestigations, such as the influence of surface pretreatments or an elastic support, are shown. Afterwards, three various synthetic bones are compared to demonstrate the possibility of the modification of modal parameters. Finally, the effect of dehydration on modal parameters is shown and discussed based on the measurement of a sheep femur.

Further research work will focus on the acquisition of modal parameters of human bones (femur). The work presented within this paper constitutes the basis for an optimal usage of rare human test structures.

\section{Author Statement}

The authors have no conflict of interest in relation to the present study. The conducted research is not related to either human or animals use. The work presented here will be co-financed with tax funds on the basis of the budget approved by the members of Parliament of Saxony (SAB FKZ 100334004, term: 04/18$12 / 20)$.

\section{References}

[1] Basso T, Klaksvik J, Syversen U, Foss OA. A biomechanical comparison of composite femurs and cadaver femurs used in experiments on operated hip fractures. Journal of biomechanics 2014;47:3898-3902

[2] Brown AD, Walters JB, Zhang YX, Saadatfar M, EscobedoDiaz JP, Hazell PJ. The mechanical response of commercially available bone simulants for quasi-static and dynamic loading. Journal of the Mechanical Behavior of Biomedical Materials 2019;90:404-416

[3] Wähnert D, Hoffmeier KL, Stolarczyk Y, Fröber R, Hofmann GO, Mückley T. Evaluation of a customized artificial osteoporotic bone model of the distal femur. Journal of biomaterials applications 2011;26:451-464

[4] Hao J, Nangunoori R, Wu YY, Rajaraman M, Cook D, Yu A, et al. Material characterization and selection for 3D-printed spine models. 3D printing in medicine 2018;4:8

[5] Neugebauer R, Werner M, Voigt C, Steinke H, Scholz R, Scherer S, et al. Experimental modal analysis on freshfrozen human hemipelvic bones employing a 3D laser vibrometer for the purpose of modal parameter identification. Journal of biomechanics 2011;44:1610-1613 